\section{THE DOCTORS' ASSOCIATION UK LEARN NOT BLAME CAMPAIGN: A CAMPAIGN FOR A JUST CULTURE IN THE NHS}

Cicely Cunningham, Jenny Vaughan, Samantha Batt-Rawden, Rinesh Parmar. The Doctors' Association UK

\subsection{6/leader-2019-FMLM.9}

The Learn Not Blame Campaign, developed by The Doctors' Association UK, was developed as a response to the anger and outrage felt in the medical profession following the January 2018 High Court decision in Bawa-Garba vs GMC, where the High Court found in favour of the GMC.

The Learn Not Blame Campaign argues for a just culture in the NHS, where each individual - patient, family member or staff - is valued and treated with respect, kindness and fairness. The campaign aims to empower individual doctors to be part of a transformational change process working towards this. The campaign encourages individuals to commit to action within their own sphere of influence, and join together as a movement to put pressure on NHS leadership.

The Learn Not Blame Campaign was launched at an event in Parliament, hosted by Dr Philippa Whitford MP and attended by the Secretary of State for Health and Social Care, Rt Hon Matt Hancock MP. The full event can be watched at https://www.youtube.com/watch?v=4C4oN_Uv29w.

The Learn Not Blame Campaign has seen significant successes, including featuring in the BMJ and mainstream national media; distributing campaign materials to individual doctors and Trusts; and maintaining a social media presence through a 10,000 strong Facebook group. The campaign was endorsed by Prof Don Berwick of the Institute for Healthcare Improvement in a video message (https://www.youtube.com/ watch?v $=\mathrm{UGbBg} 4 \mathrm{uxff}$ ).

A key challenge in developing this campaign has been to articulate - and advocate - the need for accountability of NHS organisations, including individuals working within them, after harm, within a just culture. Key to addressing this was engagement at all stages with those who have experienced avoidable harm, and ensuring that the patient/family voice is heard at all our events and throughout our campaign.

The Learn Not Blame Campaign represents what can be achieved by ordinary doctors, working with patients and families, who want to see a better NHS.

\section{THE NATIONAL PERIOPERATIVE MEDICINE ROADSHOW}

${ }^{1} \mathrm{~K}$ Samuel, ${ }^{2} \mathrm{R}$ Lal. 'North Bristol NHS Trust, UK; ${ }^{2}$ Guy's and St Thomas' NHS Foundation Trust, UK

\subsection{6/leader-2019-FMLM.10}

Perioperative medicine (POM) is a multidisciplinary specialty optimising care of high-risk surgical patients. There is a paucity of free and locally accessible POM education despite POM being an established core part of training curricula.

TRIPOM (Trainees with an Interest in Perioperative Medicine) is a voluntary multidisciplinary educational collaborative group providing free access to educational materials and events in POM. In 2018, TRIPOM launched a mobile perioperative conference,'The Perioperative Medicine Roadshow', aiming to deliver face to face education for free.

Five roadshow events have been delivered in Swansea, Brighton, Stoke, Oxford and Bristol to date. National core stakeholders in POM have been involved in planning and delivering sessions, and through central leadership with coordination involving these national bodies, along with delegation of local tasks to regional teams with excellent buy in, we have found a satellite model of team management has led to effective planning and leadership from the central team.

A total of 378 healthcare workers have attended 5 events with medical doctors, surgeons, anaesthetists and MDT members represented. Global feedback on quality of education gave a mean score of 4.41 out of 5 , with $91.8 \%$ responding that the event would improve and change their clinical practice. Live streaming online is now being used to facilitate larger audiences.

Remotely organising events has been challenging and has needed excellent communication with a clear point of contact for local teams. Use of a set event task proforma and a clear local lead has improved facilitation. Validating the course content via endorsement of Continual Professional Development points has been of benefit. Through meticulous planning, strong leadership and buy in from all parties involved, we have succeeded in leading a sustained and high quality intervention to the identified issue.

\section{THE SCOTTISH CLINICAL LEADERSHIP FELLOWSHIP PROGRAMME: A QUANTITATIVE AND QUALITATIVE EVALUATION}

Euan Harris*, Kate Arrow, Thomas Lamont. NHS Education for Scotland, UK

\subsection{6/leader-2019-FMLM.11}

Aims The Scottish Clinical Leadership Fellowship (SCLF) is a one-year programme which aims to develop future clinical leaders in NHS Scotland, within medicine and dentistry. The Fellowship is supported by NHS Education for Scotland (NES), the Scottish Government and multiple hostpartner organisations. A quantitative and qualitative evaluation of the SCLF programme was conducted to identify the key learning and reflections of participants, and to ascertain the long-term impact on Fellows upon recommencing clinical training.

Methods A total of 52 individuals, including current and all previous Fellows, were invited to complete an online questionnaire which asked participants to identify key learning from their SCLF experience, and explored their personal reflections of the Fellowship. The questionnaire also aimed to ascertain what barriers Fellows had encountered upon returning to clinical training, and how their attitudes to medical leaders and organisations had been influenced by the programme.

Results 25 individuals responded to the questionnaire. Free text comments detailing each Fellow's key learning and reflections from the SCLF programme were themed according to the NES Leadership Capabilities Framework. Fellows described an overwhelmingly positive and far-reaching impact across a 